\title{
Evaluation and Development Suggestions on Agricultural Ecotourism Resources in the Northeastern Chongqing
}

\author{
Qiong Wang \\ College of Tourism of Chongqing University of Arts and Sciences, \\ Chongqing, 402160, China
}

\begin{abstract}
To better develop agricultural tourism and ecotourism in Northeastern Chongqing. This study used AHP method to construct step evaluation model of agricultural ecotourism resources, invited experts to determine the weight of the indicator of each level and calculated the comprehensive scores by fuzzy comprehensive evaluation model. Results indicated that resource itself covered higher weight, among which the resource characteristics and resource scale are the most important indicators, The comprehensive evaluation scores show that the overall evaluation result is good, the resource's itself value is excellent, the resource environment and development conditions are good. The results suggest that we should focus on excavating the characteristics of agricultural tourism resources in the Northeastern Chongqing and expanding the scale to improve their development conditions, as well as promoting sustainable tourism development.
\end{abstract}

Keywords-Northeastern Chongqing; agro-ecological tourism resources; analytic hierarchy process; Fuzzy comprehensive evaluation; sustainable development

\section{INTRODUCTION}

Agro-ecological tourism is an important measure to protect the regional environment and promote sustainable tourism development. It is a new type of in-depth experience, which is instructed by the principle of ecology, based on agricultural production, agricultural tourism and agronomic exhibitions[1]. Many scholars have studied agro-ecological tourism. Wang Yingtao[2] and Jia Aishun[3] drew on the experience of foreign agro-ecotourism development to explore the development strategy of domestic agro-ecotourism. Some scholars researched on agro-ecological tourism resources with constructing evaluation index system[4-6], others explore the development strategy of agro-ecological tourism in a specific region through individual cases, and derived specific development strategies and reference to other types of tourism destinations[7-8]. Therefore, based on the previous evaluation methods of agro-ecotourism resources, this paper used the fuzzy comprehensive evaluation model to further comprehensively evaluate the importance of resources in the Northeastern Chongqing. The development of agro-ecological tourism in the conservation area provides theoretical support to better promote the sustainable development of agro-ecological tourism and rural tourism in the Three Gorges reservoir area. The Northeastern Ecological Conservation Development Zone includes 11 districts and counties. It is positioned as a national key ecological function zone and a major agricultural product producing area, an important ecological barrier in the Yangtze River Basin, a characteristic economic corridor in the upper reaches of the Yangtze River, an international gold tourism belt in the Three Gorges of the Yangtze River, and a special resource processing base.

\section{EVALUATION MODEL CONSTRUCTION AND ANALYSIS}

This study mainly used the Analytic Hierarchy Process (AHP) to establish a hierarchy evaluation index system. Based on the statistical results of the expert scoring method, the judgment matrix is constructed, and the weight coefficients of each index are calculated. The comprehensive evaluation of the agro-ecotourism resources in the Northeast Chongqing conservation area is carried out through the comprehensive fuzzy evaluation model.

\section{A. Model indicators selection}

According to two major categories of classification evaluation criteria, China's "Agricultural Resource Classification, Investigation and Evaluation" and "People's Republic of China Tourism Resources Classification, Investigation and Evaluation" (GBT18972-2003), combined with Zhou Wenli's approach[1], following the basic principles of "scientific, holistic and representative", combined with the on-the-spot situation of the Northeast conservation area, solicited the opinions of local agricultural bureau experts, agricultural planning experts, local tourism bureaus and relevant tourism experts from various districts and counties. After repeated discussions, the final construction was completed. The AHP evaluation index system of agro-ecotourism resources in Northeastern Chongqing is set as the total target level (A), three first-level indicators including resources itself value( A1), resources environment (A2) development conditions (A3), a total of 19 secondary indicators are established under the primary indicators. The indicators of each hierarchy are related and complemented each other, and finally, constitute the evaluation index system of agricultural ecotourism resources in Northeastern Chongqing.

\section{B. Judgment matrix Construction and inspection}

Firstly, two-two comparison judgment matrix between twolevel evaluation indicators under the construction resource itself is constructed(A11-A17). The judgment matrix P1 is 
constructed according to the importance comparison between the two indicators of A11-A17, P1=(Uij)n*n. Among them, Ui is a triangular fuzzy number. The fuzzy judgment matrices of the resource environment and development conditions are respectively recorded as $\mathrm{P} 2, \mathrm{P} 3$, and the construction method is consistent with $\mathrm{P} 1$. On this basis, the comparison judgment matrix $P$ between the first-level evaluation indicators (A1, A2, A3) is constructed, and the value of each judgment matrix is derived from the expert scoring method. The AHP model is used to generate a questionnaire, which is distributed to relevant agricultural research experts and tourism research in each district and county, and finally determines the relative importance of each indicator. In this study, 20 relevant experts are invited to fill out the AHP questionnaires the corresponding judgment matrix of each layer can be obtained. Finally, the weight of the evaluation index was calculated by the summation method. Since the score of the judgment matrix is artificially given, it is necessary to perform a consistency test on the calculation result. The study calculates the test coefficient $\mathrm{CR}$ as an index for judging the deviation of the matrix. $C I=\frac{\lambda_{\max }-n}{n-1}, C R=\frac{C I}{R I}$, Among them, The largest eigenroot of the judgment matrix $\lambda_{\max }=\frac{1}{n} \sum \frac{(P W)_{i}}{W_{i}} R I$ is the average random consistency index of the judgment matrix. If $C R \leq 0.1$, it indicates that satisfactory consistency has been obtained; otherwise, the judgment matrix needs to be further optimized until a satisfactory consistency test is obtained.

\section{Indicator weight calculation}

According to the statistical results of expert scoring, with the aid of AHP-related calculation software, the weights of the evaluation indicators at each level are obtained after optimization, and Table I is obtained.
Among the first-level indicators (A1, A2, A3), the resource itself accounts the largest proportion of the global weight, exceeding $50 \%$, which indicated that the resource itself is of great importance for the agro-ecological tourism development in the Northeastern Chongqing conservation area. Therefore, it is necessary to select the area with high resource value so that to acquire an accurate and in-depth evaluation of its own value. In the secondary evaluation index (A11-A17), the resource characteristics and resource scale account the highest weight both in the global weight and the same level weight, which are the core element of the resource's own value. It requires that when assessing the value of the agro-ecotourism resources, it should focus on the uniqueness of the resource, the characteristics of "unique" and "first" and its relative advantage. In addition, whether agricultural eco-tourism resources have formed a large scale also worth caring. At the same time, other relevant secondary evaluation indicators should also be considered carefully.

Secondly, the environment in which the resources are located, with a weight of 0.2823. Among them, the local economic development level indicator (A21) has the largest weight value in the evaluation index of the same level (A21A27), indicating that the local economic development level plays an important role in the environmental assessment of agro-ecological tourism resources in the Northeast Chongqing conservation area. In addition, other environments in which agro-ecotourism resources are located (A22-A27) cannot be ignored. Even though their weight is not high, the soundness and stability of these evaluation indicators are the basic guarantees for the development of agro-ecological tourism.

Finally, the development condition (0.1802) has the lowest weight, which has little influence, but the construction condition and traffic condition should be evaluated, which is related to the topography of Northeastern Chongqing conservation area.

TABLE I. WEIGHT VALUES OF EVALUATION INDEX SYSTEM

\begin{tabular}{|c|c|c|c|c|}
\hline Main target & Primary indicator & Secondary indicators & Global weight & Peer weight \\
\hline \multirow{19}{*}{ Evaluation of agricultural eco-tourism resources } & \multirow{7}{*}{$\begin{array}{c}\text { Resource itself } \\
0.5375\end{array}$} & Resource features a11 & 0.1446 & 0.2690 \\
\hline & & Resource scale a12 & 0.1255 & 0.2334 \\
\hline & & Resource awareness a13 & 0.0863 & 0.1606 \\
\hline & & Historical value a14 & 0.0643 & 0.1196 \\
\hline & & Cultural value a15 & 0.0487 & 0.0907 \\
\hline & & Ornamental value a16 & 0.0380 & 0.0706 \\
\hline & & Scientific research value a17 & 0.0301 & 0.0561 \\
\hline & \multirow{7}{*}{$\begin{array}{c}\text { Resource } \\
\text { environment } \\
0.2823\end{array}$} & Economic development level a21 & 0.0781 & 0.2766 \\
\hline & & Social environment a22 & 0.0550 & 0.1952 \\
\hline & & Cultural environment a23 & 0.0479 & 0.1698 \\
\hline & & Environmental stability a24 & 0.0312 & 0.1104 \\
\hline & & Disaster prevention capability a25 & 0.0306 & 0.1083 \\
\hline & & Environmental self-purification ability a26 & 0.0213 & 0.07 v 54 \\
\hline & & Environmental carrying capacity a27 & 0.0182 & 0.0643 \\
\hline & \multirow{5}{*}{$\begin{array}{c}\text { Development } \\
\text { condition } \\
0.1802\end{array}$} & Construction conditions a31 & 0.0668 & 0.3706 \\
\hline & & Traffic conditions a32 & 0.0488 & 0.2709 \\
\hline & & Source market a33 & 0.0265 & 0.1468 \\
\hline & & Tourist facilities a34 & 0.0201 & 0.1114 \\
\hline & & Community resident attitude a35 & 0.0180 & 0.1003 \\
\hline
\end{tabular}




\section{THE FUZZY COMPREHENSIVE EVALUATION OF AgriculturAl ECO-TOURISM RESOURCES IN NORTHEASTERN CHONGQING CONSERVATION AREA}

\section{A. Model Overview}

The comment level domain $\mathrm{V}=(\mathrm{v} 1, \mathrm{v} 2, \mathrm{v} 3, \mathrm{v} 4)=($ excellent, good, medium, poor) is established, and each comment level can correspond to a fuzzy subset. After constructing the level fuzzy subset, one by one The evaluation elements are quantified from each index, and the membership degree of the superior evaluation index to the hierarchical fuzzy subset is determined from the evaluation factors, and the fuzzy relation matrix $\mathrm{Ri}_{*_{\mathrm{j}}}$ is obtained, In the matrix $\mathrm{R}_{\mathrm{i}^{*} \mathrm{j}}$, the $\mathrm{i}$-th row and the $\mathrm{j}$ th column element rij represent the membership degree of the Vjlevel fuzzy subset from the index ui. Using the principle of maximum subordination in fuzzy statistics, the agro-ecological tourism resources in the ecological conservation area of Northeastern Chongqing were graded and evaluated, and the final evaluation results were obtained.

\section{B. Data calculation}

Based on the indicators of the evaluation system, the questionnaire was designed. The questionnaire is based on the Likert scale, and each evaluation index is divided into four levels, and the values are assigned to 4, 3, 2, 1, representing excellent (E1), good (E2), medium(E3), and poor(E4).

The questionnaire was distributed to experts who are majored in agriculture and tourism research, scenic development and management, local residents were also participated. Almost200 questionnaires were distributed and 198 were recovered. The recovery rate was 99\%, 195 were valid questionnaires, and the effective rate was $98 \%$. According to the results of the questionnaire, using the $\mathrm{M}\left({ }^{*},+\right)$ operator and the weighted average calculation rule, the $\mathrm{W}$ is combined with the $\mathrm{R}$ of each evaluated index to calculate the fuzzy comprehensive evaluation value of each level of evaluation indicators. Among them, the resource selfevaluation vector (R1), the resource environment fuzzy evaluation vector (R2), the development condition fuzzy evaluation vector is $\mathrm{R} 3$.

According to the above calculation results, the comprehensive score of the secondary indicator system is: $\mathrm{VA} 1=3.0479, \mathrm{VA} 2=2.7841, \mathrm{VA} 3=2.6107$. According to the above calculation results, the comprehensive evaluation matrix of agro-ecotourism resources in the Northeastern reclamation area is obtained, $\mathrm{VA}=2.8946$.

$$
\begin{aligned}
& \mathbf{R}=\left[\begin{array}{llll}
0.3791 & 0.3177 & 0.2752 & 0.0280 \\
0.2107 & 0.3953 & 0.3614 & 0.0326 \\
0.1545 & 0.3756 & 0.3960 & 0.0739
\end{array}\right] \\
& \mathbf{O}=\mathbf{W} * \mathbf{R}=\left[\begin{array}{llll}
0.2911 & 0.3500 & 0.3213 & 0.0376
\end{array}\right]
\end{aligned}
$$

\section{Data Analysis}

The resource's itself value (A1) has the highest comprehensive score (3.0479), it is E1 level, and its comprehensive fuzzy evaluation result is excellent. It shows that the overall value of agro-ecological tourism resources in Northeastern Chongqing is high. The comprehensive scores of resource environment and development conditions are 2.7841 and 2.6107, respectively, all of which is E2. The comprehensive fuzzy assessment results are good, indicating that the agro-ecological tourism resources in the Northeastern Chongqing Conservation Area are currently in a good environment with good development conditions. The overall score of agro-ecological tourism resources in the Northeastern reclamation area is 2.8946, with a grade of E2. The comprehensive assessment results are good, indicating that the overall quality of agro-ecotourism resources in the Northeastern reclamation area is better, but further improvements are needed in many aspects.

\section{RESEARCH CONCLUSIONS AND RECOMMENDATIONS}

\section{A. Research conclusions}

The resource value and resource scale have the highest important value, and the weight in the same level indicator also reflects the consistency, indicating that the resource characteristics and resource scale are the core and foundational element for the development of the agricultural eco-tourism in the Northeastern conservation area. The fuzzy comprehensive evaluation model is used to further comprehensively evaluate the weight. The overall evaluation result is good, indicating that the Northeastern reclamation area has a good resource base for developing agro-ecological tourism. Among them, the evaluation result of the resource's own value is excellent, and the other two indicators are good. It is indicated that the agroecological tourism resources in Northeastern Chongqing have better resource characteristics and larger resources. The comprehensive value of agricultural historical value, agricultural ornamental value and cultural value is higher. At the same time, the environment and development conditions of its resources are also relatively good. This evaluation result is consistent with the distribution of weight values in the evaluation index system, which verifies the reliability and practicability of the analysis conclusions, which will promote the development of agro-ecological tourism throughout the Northeastern Chongqing Conservation Area, and also for the entire Three Gorges reservoir area. Tourism lays the foundation.

\section{B. Development Suggestions}

First, in the process of agro-ecological tourism development, we should focus on the value of agro-ecological tourism resources, analyze the greatest characteristics of the resources themselves, and vigorously develop and build them. The highlight of the resource itself is made into a characteristic agro-ecological tourism product, which is used as an attraction point. Based on a certain agro-ecological tourism product, it will drive the development and development of the surrounding tourism industry, thus forming a scale effect. Second, we should focus on analyzing the social environment in which resources are located, strengthen the cultural atmosphere, integrate local unique folk culture into the development process of agro-ecological tourism, and increase the functionality and attractiveness of agro-ecological tourism products.in the 
development of agro-ecological tourism products in Northeastern Chongqing, it is necessary to strengthen the protection of the environment, improve the environmental disaster prevention capabilities, and develop related products within the scope of the self-purification capacity of the surrounding environment and its carrying capacity. Create a sound environment. At last, it is very important to pay attention to the on-site development environment of various districts and counties in the Northeastern conservancy area, and to develop agro-ecological tourism in an appropriate way, and to combine the actual conditions to create resource development conditions. According to the different geographical locations of the various villages in each district and county, targeted and differentiated development is carried out, and finally, each has its own characteristic agricultural eco-tourism development model.

\section{REFERENCES}

[1] Wang Cuiping. An empirical study on the evaluation system of agroecological tourism resources. China Agricultural Resources and Regionalization, 2018, 39(1): 66-70.(In Chinese)

[2] Wang Yingtao. Experiences and References on the Development of World Agro-ecological Tourism [J]. Reform and Strategy, 2018, 34(3) 112-114.(In Chinese)

[3] Jia Aishun. Research on the Coordinated Development of Agroecological Tourism and Economy [J]. Agricultural Economy, 2018 (8) 23-24.

[4] Lin Xiuzhi, Chen Qiuhua, Lai Qifu. Research on the Construction of Agricultural Eco-tourism Resources Evaluation Model[J].Forestry Economics, 2010, 30(6): 507-515.(In Chinese)

[5] Yu Youzhen, Wang Wei. Research on the Construction of Evaluation Indexes of Urban Agricultural Eco-tourism Resources[J].Shanxi Agricultural Journal,2016(8):6-7.(In Chinese)

[6] Li Ying. Construction of Agricultural Eco-tourism Resources Evaluation Index System in Zhejiang Province [J]. China Agricultural Resources and Regionalization, 2018, (In Chinese)

[7] Qiao Jie, Leteng. Analysis on the development status and countermeasures of eco-agricultural tourism in Hebei Province[J].Ecology and Economy,2016,32(2):132-137.(In Chinese)

[8] Zhu Wanchun, Liao Junhua. Analysis of Geographical Advantages and Regional Characteristics of Agricultural Eco-tourism in Guizhou Province [J]. China Agricultural Resources and Regional Planning, 2018 39(9):304-308. (In Chinese) 